\title{
Individual differences in the Behavioral Inhibition System are associated with orbitofrontal cortex and precuneus gray matter volume
}

\author{
Paola Fuentes • Alfonso Barrós-Loscertales • \\ Juan Carlos Bustamante • Patricia Rosell • \\ Víctor Costumero • César Ávila \\ Published online: 17 May 2012 \\ (C) Psychonomic Society, Inc. 2012
}

\begin{abstract}
The Behavioral Inhibition System (BIS) is described in Gray's Reinforcement Sensitivity Theory as a hypothetical construct that mediates anxiety in animals and humans. The neuroanatomical correlates of this system are not fully clear, although they are known to involve the amygdala, the septohippocampal system, and the prefrontal cortex. Previous neuroimaging research has related individual differences in BIS with regional volume and functional variations in the prefrontal cortex, amygdala, and hippocampal formation. The aim of the present work was to study BIS-related individual differences and their relationship with brain regional volume. BIS sensitivity was assessed through the BIS/BAS questionnaire in a sample of male participants $(N=114)$, and the scores were correlated with brain regional volume in a voxel-based morphometry analysis. The results show a negative correlation between the BIS and the volume of the right and medial orbitofrontal cortices and the precuneus. Our results and previous findings suggest that individual differences in anxiety-related personality traits and their related psychopathology may be associated with reduced brain volume in certain structures relating to emotional control (i.e., the orbitofrontal cortex) and self-consciousness (i.e., the precuneus), as shown by our results.
\end{abstract}

Keywords Behavioral Inhibition System · Personality · Anxiety · Prefrontal cortex · Orbitofrontal cortex ·

Precuneus · Voxel-based morphometry

Paola Fuentes and Alfonso Barrós-Loscertales contributed equally to this work.

P. Fuentes $(\varangle) \cdot$ A. Barrós-Loscertales · J. C. Bustamante •

P. Rosell $\cdot$ V. Costumero $\cdot$ C. Ávila

Departamento de Psicología Básica,

Clínica y Psicobiología, Universitat Jaume I,

Av. de Vicent Sos Baynat, s/n 12071,

Castelló de la Plana, Spain

e-mail: pfuentes@uji.es
In Gray's Reinforcement Sensitivity Theory (RST, Gray, 1982), behavior is thought to be mediated by the activity of three motivational systems: the fight-flight system (FFS), which is sensitive to unconditioned aversive, painful stimuli; the behavioral activation system (BAS), which responds to reward and to relief of punishment; and the behavioral inhibition system (BIS), which is sensitive to conditioned aversive stimuli, punishment signals, and termination of reward. The revision of the theory (Gray \& McNaughton, 2000) proposed a change for the role of the BIS, which was made responsible for the resolution of conflicts between the BAS (now sensitive to conditioned and unconditioned rewarding stimuli) and the FFS (now responding to conditioned and unconditioned aversive stimuli).

BIS activity results in the suppression of ongoing behavior and enhancement of attention and information processing. Along with the inhibition of dominant behaviors and the engagement of risk-assessment processes, BIS activity results in emotional states of anxiety, subjectively experienced as worry and rumination, and a sense of possible danger/loss (Corr, 2004). Thus, differences in the sensitivity of the BIS result in individual differences in the anxiety trait. Extremely high or low levels of BIS sensitivity are also related to pathologies. An overactive BIS is related to clinical conditions of anxiety (Kimbrel, 2008; Vervoort et al., 2010) and affective disorders (Hundt, Nelson-Gray, Kimbrel, Mitchell, \& Kwapil, 2007; Kasch, Rottenberg, Arnow, \& Gotlib, 2002; Pinto-Meza et al., 2006; Sportel, Nauta, Hullu, de Jong, \& Hartman, 2011), whereas a hypoactive BIS leads to risk proneness and is related to (primary) psychopathy (Corr, 2008).

When determining the neural substrates of the BIS, Gray (1977; Gray \& McNaughton, 2000; for a review see Corr, 2004) observed the action of anxiolytic drugs in animal samples. Gray and McNaughton proposed that cortical and subcortical structures are involved in the sensitivity of the 
BIS, with a prominent role for the amygdala and the septohippocampal system, along with regions of the cingulate and the prefrontal cortex. At the structural level, researchers in previous studies have related individual differences in BIS sensitivity with variations in the regional volume of the amygdala, the hippocampus, and the parahippocampal areas in that greater scores in the different BIS-sensitivity measures were associated with an increased volume in these structures (Barrós-Loscertales et al., 2006a; Cherbuin et al., 2008). Other BIS-related personality traits have also been linked to structural variation in the brain. For example, the trait named harm avoidance was defined by Cloninger (1986) as the individual tendency to inhibit behaviors, and it has been shown to be a good predictor of the BIS (Mardaga \& Hansenne, 2007). Gardini, Cloninger, Robert, and Venneri (2009) found a negative correlation between harm avoidance and the volume of the middle and inferior frontal gyri, the cuneus, and the precuneus, as well as other parietal and occipital areas. Likewise, the five-factor model's (Costa \& McCrae, 1992) neuroticism trait is closely related to BIStrait measures (Smits \& Boeck, 2006). Wright et al. (2006) noted that individual differences in neuroticism were negatively correlated with the regional cortical thickness of the orbitofrontal and medial frontal cortex. On the other hand, DeYoung et al. (2010) found a wide range of brain regions in which local volume was associated with neuroticism in a structural MRI study. Neuroticism correlated positively with the brain volume in the cingulate cortex and in the middle temporal lobe and negatively with the volume of right dorsolateral prefrontal cortex, portions of the medial temporal, the basal ganglia, and the midbrain (DeYoung et al., 2010).

Measurement of BIS sensitivity has been usually accomplished by questionnaire, although a substantial number of the scales that have been used were indirect measures developed under other theoretical frameworks than Gray's theory (Caseras, Ávila, \& Torrubia, 2003; see Torrubia, Ávila, \& Caseras, 2008, for a review). Behavioral measures such as the startle reflex have also been shown to be sensitive to this trait. High scores in the BIS scales are associated with a potentiation of the startle reflex in the affective startle reflex modulation (ASRM) paradigm (Montag et al., 2008). Currently, the most widely used measure for BIS sensitivity is the BIS scale of the BIS/BAS questionnaire (Carver \& White, 1994) and, to a lesser extent, the Sensitivity to Punishment scale of the SPSRQ (Sensitivity to Punishment and Sensitivity to Reward Questionnaire; Torrubia, Ávila, Molto \& Caseras, 2001). Both were developed on the basis of the Reinforcement Sensitivity Theory (RST). In the present study, the BIS/BAS questionnaire was used. It should be noted that this scale was developed within the original RST framework and not the revised version of Gray and McNaughton (2000). Thus, it intends to measure the sensitivity of the BIS as originally defined by Gray (1982) as a punishment and passive avoidance system. However, as there is an overlap between the old and the new conception of the BIS, the Carver and White's BIS scale might still be a reliable measure of the revised BIS construct (Berkman, Lieberman, \& Gable, 2009). The BIS subscale comprises seven items that reflect a concern about possible aversive events or sensitivity to such events when they occur. According to Carver and White (1994), the "BIS scale was designed to measure sensitivity to anxiety provoking stimuli rather than ambient affective tone," a feature that was informed to be different for other related scales. Although a considerable amount of studies has shown that different BIS-sensitivity scales and other related measures (e.g., harm avoidance, neuroticism, etc.) are strongly correlated, they also present a considerable degree of uncommon variance (see Torrubia et al., 2008). Future research should serve to determine subtle yet possible differences between these measures.

The aim of our present work was to study individual differences in BIS sensitivity and their relationship with regional brain volume using a voxel-based morphometry analysis. The BIS scores were expected to correlate with the regional volume changes in brain regions previously identified as relevant for this trait, such as the prefrontal regions, the amygdala, and the hippocampus.

\section{Materials and methods}

\section{Participants}

BIS sensitivity was assessed in a sample of 114 male participants ( $M_{\text {age }}=26.76 ; S D=7.48$; age range: $18-53$ years). All the participants completed the BIS scale from the BIS/ BAS questionnaire (Carver \& White, 1994). Two of the participants were left-handed, whereas the other 112 were right-handed according to the Edinburgh Handedness Inventory (Bryden, 1977; Oldfield, 1971). None of the participants had a previous psychiatric or neurologic diagnosis. The study was approved by the Ethical Committee of the Universitat Jaume I, and all the participants signed a written informed consent.

\section{MRI Acquisition}

Images were acquired with a $1.5 \mathrm{~T}$ Siemens Avanto scanner (Erlangen, Germany). High-resolution whole-brain 3-D images were acquired using a T1-weighted gradient echo pulse sequence (TR, $11 \mathrm{~ms}$; TE, $4.9 \mathrm{~ms}$; flip angle, $90^{\circ}$; voxel size, $1 \mathrm{~mm}^{3}$; matrix, $224 \times 256 \times 176 \mathrm{~mm}$ ). 
Image Preprocessing

Voxel-based morphometry (VBM) was performed with the VBM8 toolbox (http://dbm.neuro.uni-jena.de/vbm/) for the SPM8 package (Wellcome Department of Imaging Neuroscience, London; http:/www.fil.ion.ucl.ac.uk/spm/ software/spm8/). The preprocessing procedure implemented in VBM8 is based on the unified segmentation approach, which combines segmentation, normalization, and bias correction in a unified model (Ashburner \& Friston, 2005). Images were segmented into gray matter, white matter and cerebrospinal fluid, and were registered by affine (12-parameter) transformations to a standard template provided by the International Consortium of Brain Mapping (ICBM). Further spatial registration was achieved by high-dimensional Dartel normalization. Voxel values were modulated by the nonlinear components derived from spatial normalization; thus, the volume variations resulting from normalization were corrected in the resulting gray matter volume maps. Modulation by linear components was not performed in order to account for individual differences in global brain size in the subsequent analyses (http://dbm.neuro.uni-jena.de/vbm/segmentation/ modulation/), thus rendering correction for total intracranial volume of the individual unnecessary (Scorzin et al., 2008). Hence, inferences were made on local gray matter volume changes. Finally, images were smoothed with a 12-mm Gaussian kernel.

\section{Statistical analysis}

BIS sensitivity was correlated with the gray matter volume in a voxel-by-voxel regression analysis with scores on the BIS scale as the covariate of interest within the general linear model framework in SPM8. The parameters to determine the regions of significant differences were set at $p<.05$, FWE-corrected for multiple comparisons at the cluster level using an auxiliary (uncorrected) voxel threshold of $p<.001$. The corrected extent threshold (cluster size $k>1223$ ) was defined with the algorithm implemented in the CorrClusTh program by Thomas Nichols (http://www2.warwick.ac.uk/fac/sci/statistics/staff/ academic-research/nichols/scripts/ spm/johnsgems5/ \#Gem6) to allow the FWE-correction at the cluster level. In order to control the effect of potential confounding variables, a second multiple regression model was created by adding age as a covariate. Although this goes beyond the scope of the present study, we also conducted similar analyses for the BAS subscales and a combined BAS measure, which resulted from the addition of the three BAS subscales.

In addition to the whole-brain analysis, the association between the BIS scores and the gray matter volume in the hypothesized discrete structures was studied by the definition of a priori regions of interest (ROIs) in the hippocampus and the amygdala. These ROIs were defined using the Automated Anatomical Labeling Atlas (Tzourio-Mazoyer et al., 2002) included in the WFU Pickatlas software (Maldijan, Laurienti, Kraft, \& Burdette, 2003). The first eigenvariate of each ROI was extracted as a measure of its average signal and was correlated with the BIS scores $(p<.05)$.

\section{Results}

Personality scores

All the participants $(N=114)$ completed the BIS/BAS questionnaire (Carver \& White, 1994). The mean scores and standard deviations of the BIS scale and the BAS subscales are detailed in Table 1 and the correlation matrix is shown in Table 2. The scores obtained are similar to those observed in the original standardization of the scale (Carver \& White, 1994) and in previous reports using the same measure (Caseras et al., 2003).

\section{Voxel-Based Morphometry (VBM)}

The VBM whole-brain analysis $(p<.05$ FWE-corrected at the cluster level, $k$-threshold $>1223$ ) showed two clusters of gray matter that correlated negatively with the BIS scores (Fig. 1). The first cluster was located at the precuneus $(k=4077)$, involving bilateral regions with the local maxima $(T=4.82)$ in the left hemisphere $(x=-2, y=-75, z=46)$. The second cluster $(k=2726)$ extended from the medial orbitofrontal cortex $(T=4.07$, coordinates $x=-11, y=50, z=-15)$, to its local statistical maxima $(T=4.21)$ at the right lateral orbitofrontal cortex (coordinates $x=39, y=36, z=-12$ ). No significant positive correlations were found. The three BAS subscales and the combined BAS score did not show any significant positive or negative correlation with the gray matter volume at the predefined statistical and extent thresholds.

An additional analysis was performed to study the effect of age on the present results given previous reports showing the effects of this kind of nuisance covariates ( $\mathrm{Hu}$ et al.,

Table 1 Mean scores, standard deviations, and Cronbach's alpha for each subscale

\begin{tabular}{llll}
\hline Scale & $M$ & $S D$ & Alpha \\
\hline BIS & 20.28 & 3.49 & .73 \\
BAS-drive & 10.78 & 2.10 & .72 \\
BAS-reward responsiveness & 17.23 & 1.98 & .66 \\
BAS-fun seeking & 12.06 & 2.04 & .59 \\
BAS (combined measure) & 27.17 & 4.81 & .79 \\
\hline
\end{tabular}


Table 2 Correlations among the BIS and BAS subscales, and age $(* p<.05, * * p<.01)$

\begin{tabular}{|c|c|c|c|c|c|}
\hline & BIS & $\mathrm{D}$ & $\mathrm{RR}$ & FS & Age \\
\hline BIS & & $-.283^{* *}$ & -.008 & -.129 & $.221 *$ \\
\hline $\mathrm{D}$ & & & $.479 * *$ & $.367 * *$ & $-.240 *$ \\
\hline $\mathrm{RR}$ & & & & $.456^{* *}$ & -.036 \\
\hline FS & & & & & $-.229 *$ \\
\hline
\end{tabular}

$D$ Drive; $R R$ reward responsiveness; $F S$ fun seeking

2010). When using age as a covariate, the VBM analysis showed the same clusters of negative correlation, although these results did not survive the FWE correction at the cluster level and were only significant with an uncorrected statistical threshold of $p<.001$. As in the previous analysis, a negative correlation was found at the precuneus $(T=4.11$, coordinates $x=0, y=-76, z=46$ ) with an extension of 894 voxels. The orbitofrontal cortex showed two negatively correlating clusters, the first located at the right hemisphere $(T=3.41$, coordinates $x=38, y=35, z=-12)$ with an extension of 54 voxels, and the second at the medial orbitofrontal cortex $(T=3.36$, coordinates $x=-11, y=50, z=-15)$ with 71 voxels; both included the same regions reported before correcting for age effects. Another cluster of negative correlation was found at the right parietal cortex $(T=4.13$, coordinates $x=-29, y=-70, z=57$ ) with an extension of 417 voxels, which was not present before. However in our sample age and BIS scores were significantly correlated $(r=$ $.22, p<.05)$. It is likely that modeling out age effects will also partially model out the effects of the BIS scores because of a certain degree of collinearity effect. Therefore, we will focus on the discussion of former results without forgetting the potential effects of age. Otherwise, the ROI analysis did not show any significant correlation between the BIS scores and the amygdalar or hippocampal volumes.

\section{Discussion}

The results of the present study show the morphometric correlates of the BIS scale of the BIS/BAS questionnaire in a large sample of male participants. Specifically, our results show that greater BIS-sensitivity is associated with a reduced regional volume of the right orbitofrontal cortex (OFC) and the precuneus.

According to the RST, we hypothesized that individuals' BIS sensitivity would have been associated with structural
Fig. 1 Regional gray matter volume showing negative correlations with BIS scores $(p<.05$ FWE-corrected at the cluster level). The right-hand side of the image is the righthand side of the brain. The color bar depicts $\mathrm{T}$ values. The scatter plots show the correlations with the regional gray matter volume at the local maxima of each region (the lines correspond to the regression slope)
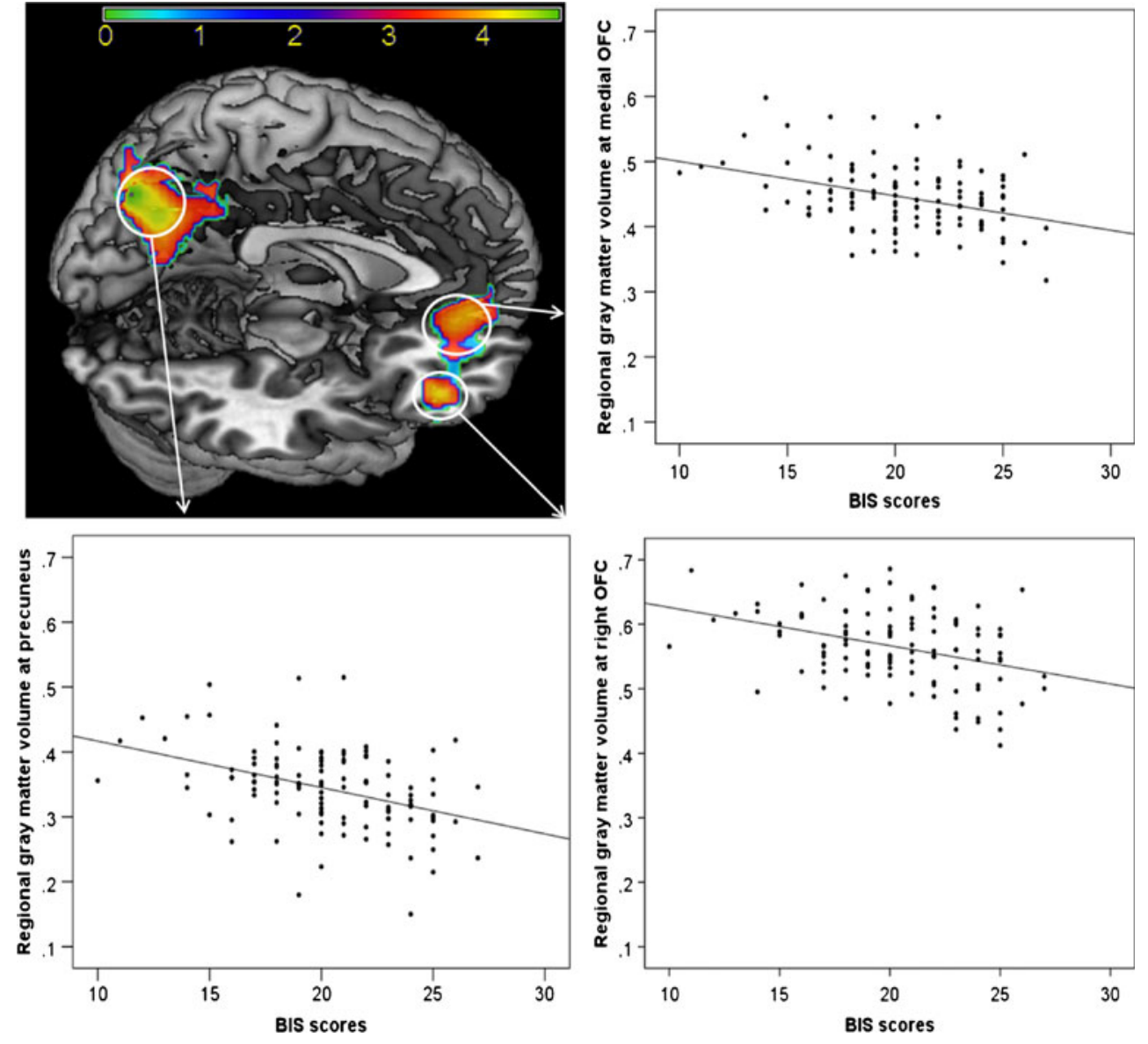
changes in the prefrontal cortex, the hippocampus, and the amygdala. The present results show that BIS sensitivity is associated with reduced gray matter in the OFC and the precuneus, but no significant associations were found between the BIS scores and the gray matter volume in the hippocampus and the amygdala. There are no previous studies employing the BIS scale that present this relationship, but there are some reporting comparable results with BIS-related traits. Cloninger's temperamental dimension harm avoidance has been seen to be negatively related to the regional volume at the anterior cingulate cortex (Van Schuerbeek, Baeken, De Raedt, De Mey, \& Luypaert, 2011), the right OFC (Gardini et al., 2009), and left precuneus (Gardini et al., 2009). In the same vein, high neuroticism scores have been associated with a reduced cortical thickness of the OFC in healthy subjects (Wright et al., 2006). Because neuroticism is a BIS-related trait, this result points in the same direction as that reported in the present study.

Besides healthy individuals, structural abnormalities in the OFC have been found in patients with affective (Bremner et al., 2002; Lacerda et al., 2004) and anxiety disorders (Hayano et al., 2009; Roppongi et al., 2010; Sobanski et al., 2010). A reduced regional brain volume in the OFC has been related to reduced gyrification and anxiety-related traits in panic disorder (Roppongi et al., 2010). Roppongi et al. suggested that given the lack of differences in gyrification between patients with panic disorders and healthy controls, anxiety-related volume changes in the OFC could be associated with neurodevelopment in these patients rather than with other factors associated with their clinical condition, such as aversive life events. This suggestion may involve the known association between BIS-related traits and emotional disorders.

Our results also reveal an unexpected decrease in precuneus volume associated with higher BIS sensitivity. It has been suggested that this structure is an important component of the interwoven network of the neural correlates of selfawareness and consciousness (see Cavanna \& Trimble, 2006, for a review). Although not part of our initial hypothesis, precuneus volume has been previously related to a BISrelated trait such as Harm Avoidance (Gardini et al., 2009) and showed a negative correlation, as in the present study. An association has also been found between neuroticism, another BIS-related trait, and the precuneus function. Neuroticism has been related to threat perception and to reduced activity of the anterior and posterior cingulate cortices, the precuneus, and temporal areas including the hippocampus and the putamen, the thalamus and the middle occipital gyrus (Kumari et al., 2007). Previous studies have shown connectivity between the OFC and the amygdala (Holland \& Gallagher, 2004; Zald \& Kim, 1996; Hahn et al., 2011), and also between the precuneus and the amygdala (Hahn et al., 2011). These results suggest a modulatory role for both OFC and the precuneus over amygdala activity, which could be related to individual differences in the Anxiety trait since amygdala activity has been shown to be related to anxiety in patients with anxiety disorders (Hahn et al., 2011; Phan, Fitzgerald, Nathan, \& Tancer, 2006) and in healthy subjects (Haas, Omura, Constable, \& Canli, 2007).

Although we hypothesized an association between BIS sensitivity and the amygdalar and hippocampal volume, our results showed no such association. Theoretically developed measures of BIS personality traits, such as sensitivity to punishment (Torrubia et al., 2001), presented a positive correlation between this trait and the regional volume of the amygdala and hippocampus in a previous study (Barrós-Loscertales et al., 2006a), but did not show an association with the OFC. Similar results were obtained with the BIS scale from the BIS/BAS questionnaire using a volumetric region of interest-based analysis instead of VBM in the hippocampus (Cherbuin et al., 2008). On the other hand, other BIS-related traits have revealed contrary results. The anxiety trait from the STAI-T has been associated with decreased volume of the amygdala and the parahippocampal areas (Hayano et al., 2009; Spampinato, Wood, De Simone, \& Grafman, 2009). Besides, harm avoidance has shown decreased hippocampal volumes associated with its related individual differences in wide sample studies (Yamasue et al., 2008), as well as positive correlations with the left amygdala volume (lidaka et al., 2006). However, it is worth noting that Harm Avoidance has shown sex differences in its amygdala association. In the study by Iidaka et al. (2006), only the female group presented an association between harm avoidance and amygdala volume, which contrasts with the present and previous (Barrós-Loscertales et al. 2006a) studies, performed with a male sample. Therefore, although the amygdala and hippocampus have been consistently associated with anxiety at the functional level (Frings, Schulze-Bonhage, Spreer, \& Wagner, 2009; Hahn et al., 2011; Haas et al., 2007; Kim \& Whalen, 2009; Phan et al., 2006; Rauch, Shin, \& Wright, 2003), structural studies offer mixed results, which might be partly expected from the use of different samples and questionnaires as we discuss below. On the other hand, differences relating to the VBM procedures could partly explain the lack of association between the BIS scores and amygdalar and hippocampal volumes because the image preprocessing used in VBM8 differs from the optimized VBM proposed by Good et al. (2001), which was used in previous studies (Barrós-Loscertales et al., 2006a).

The results of the present work are also relevant to understand the nature of the different BIS-related measures. Lack of agreement with other studies, which found differences in these structures, could be partly related to the different personality measure used in each case. The BIS/ BAS questionnaire used in the present study was developed 
under the RST framework (Caseras et al., 2003; Carver \& White, 1994), but differs conceptually from other BIS-related scales since the BIS/BAS items focus on experience of anxiety per se and not on its behavioral outcomes, which are dependent not only on BIS-sensitivity, but also on many other factors such as the ability to cope with the anxiety-provoking situation (Carver \& White, 1994). Moreover, this measure was developed within the original RST framework (Gray, 1982), which postulated a central role for the septohippocampal system in BIS functioning, but did not establish a role for the amygdala. The revised version (Gray \& McNaughton, 2000) defined the importance of the amygdala and the hippocampus and established differential roles for them in fear and anxiety (McNaughton \& Corr, 2004). We hypothesized that amygdalar and hippocampal volumes would be related to the BIS scores in the light of previous research showing this relationship. The BIS scale is likely to be a mixed measure of fear and anxiety rather than only the anxiety trait (Jackson, 2009). A more accurate distinction between fear and anxiety could lead to the observation of clearer relationships between them and the amygdalar and hippocampal volumes. The lack of specificity of the BIS scale should be acknowledged as a limitation in the present study and others using the same measure. Future works may use more specific measures since some efforts have been made in building scales under the revised RST framework, such as the Jackson-5 scales (Jackson, 2009), although their use has not yet been generalized.

An analysis of the BAS subscales was also conducted, although it was not the objective of this study. The three BAS subscales and the combined measure resulting from adding the subscales scores did not show any positive or negative correlation at the established statistical and extent thresholds. This result is not in agreement with our previous study in which sensitivity to reward, a BAS-related measure, showed a negative association with the striatum gray matter volume (Barrós-Loscertales et al., 2006b). This result could be explained by the defined statistical threshold, which may have been too restrictive to allow the BAS-related correlations to reach significance. By lowering the statistical threshold to $p<.001$ (uncorrected, minimum cluster extent of 100 voxels), a positive correlation between the drive subscale and the right fusiform gyrus was observed. However, no other significant associations were found. The lack of replication of previous results could also be related with the different personality measure used in each case, the BAS subscales and the sensitivity to reward scale. These measures have shown moderate positive correlations in previous studies and were designed to tap into the same construct. However, they may have some different psychometric properties since a high heterogeneity among the BAS measures has been suggested (Caseras et al., 2003). More research is needed to fully understand the associations between the personality traits proposed by the RST and brain structure.
Otherwise, the present study has several limitations that need to be considered. The present results have been extracted from a male sample, so it is not possible to generalize them to the entire population since prior studies have reported structural differences in orbitofrontal cortex between men and women (Gur, Gunning-Dixon, Bilker, \& Gur, 2002; Welborn et al., 2009). Thus, future research on the neuroanatomical correlates of personality will benefit from the study of both male and female samples. Another limitation of the present study is the relative effect of age on the associations observed between brain structure and the BIS scores. Accounting for the effects of age in our statistical model resulted in a lower statistical significance of the reported results. However, a relative effect of collinearity found between age and the BIS scores made it difficult to fully disentangle the actual effect of age on the reported associations. Nevertheless after taking into account the effects of age, the regional volume of the same regions was seen to be significantly associated with the BIS scores at a lower extent threshold, but which was similar to previous reports (Blankstein, Chen, Mincic, McGrath, \& Davis, 2009; Iidaka et al., 2006; Spampinato et al., 2009; Van Schuerbeek et al., 2011). Another issue entails the relationship between structure and function. Since this is a structural study, the functional implications of the regions associated with BIS sensitivity remain unclear.

In summary, our results show a decrease in the OFC and the precuneus volumes associated with higher BIS scores. The structural differences in them both could reflect individual differences in their functioning, although our results do not allow us to infer the nature of these functional variations. However, on the basis of previous findings for the activity of these structures, these results suggest that they may play a role in the modulation of anxiety, thus giving rise to individual differences in the anxiety trait.

Author note This research was supported by grants from FEPAD (Fundación para el Estudio, Prevención y Asistencias a la Drogodependencia), from Universitat Jaume I-Bancaja (P1-1B2008-35 and P11A2010-01) and from the funding agency CONSOLIDER-INGENIO 2010 Program (CSD2007-00012).

Correspondence concerning this article should be addressed to Paola Fuentes, Department of Psychology, Universitat Jaume I, Av. de Vicent Sos Baynat, s/n 12071 Castelló de la Plana, Spain (e-mail: pfuentes@uji.es).

\section{References}

Ashburner, J., \& Friston, K. J. (2005). Unified segmentation. NeuroImage, 26, 839-851. doi:10.1016/j.neuroimage.2005.02.018

Barrós-Loscertales, A., Meseguer, V., Sanjuán, A., Belloch, V., Parcet, M. A., Torrubia, R., \& Ávila, C. (2006a). Behavioral Inhibition System activity is associated with increased amygdala and hippocampal 
gray matter volume: A voxel-based morphometry study. NeuroImage, 33, 1011-5. doi:10.1016/j.neuroimage.2006.07.025

Barrós-Loscertales, A., Meseguer, V., Sanjuán, A., Belloch, V., Parcet, M. A., Torrubia, R., \& Ávila, C. (2006b). Striatum gray matter reduction in males with an overactive behavioral activation system. European Journal of Neuroscience, 24(7), 2071-4. doi:10.1111/j.1460-9568.2006.05084.x

Berkman, E. T., Lieberman, M. D., \& Gable, S. L. (2009). BIS, BAS, and response conflict: Testing predictions of the revised reinforcement sensitivity theory. Personality and Individual Differences, 46(5-6), 586-591.

Blankstein, U., Chen, J. Y. W., Mincic, A. M., McGrath, P. A., \& Davis, K. D. (2009). The complex minds of teenagers: Neuroanatomy of personality differs between sexes. Neuropsychologia, 47, 599-603.

Bremner, J. D., Vythilingam, M., Vermetten, E., Nazeer, A., Adil, J., Khan, S., Staib, L. H., et al. (2002). Reduced volume of orbitofrontal cortex in major depression. Biological Psychiatry, 51, 273-9.

Bryden, M. P. (1977). Measuring handedness with questionnaires. Neuropsychologia, 15, 617-624

Carver, C. S., \& White, T. L. (1994). Behavioral inhibition, behavioral activation, and affective responses to impending reward and punishment: The BIS/BAS Scales. Journal of Personality and Social Psychology, 67, 319-333. doi:10.1037/0022-3514.67.2.319

Caseras, X., Avila, C., \& Torrubia, R. (2003). The measurement of individual differences in Behavioural Inhibition and Behavioural Activation Systems: A comparison of personality scales. Personality and Individual Differences, 34, 999-1013. doi:10.1016/ S0191-8869(02)00084-3

Cavanna, A. E., \& Trimble, M. R. (2006). The precuneus: A review of its functional anatomy and behavioural correlates. Brain: A journal of neurology, 129, 564-83. doi:10.1093/brain/aw1004

Cherbuin, N., Windsor, T. D., Anstey, K. J., Maller, J. J., Meslin, C., \& Sachdev, P. S. (2008). Hippocampal volume is positively associated with behavioural inhibition (BIS) in a large community-based sample of mid-life adults: The PATH through life study. Social Cognitive and Affective Neuroscience, 3, 262-9. doi:10.1093/scan/nsn018

Cloninger, C. R. (1986). A unified biosocial theory of personality and its role in the development of anxiety states. Psychiatric Developments, 4, 167-226.

Corr, P. J. (2004). Reinforcement sensitivity theory and personality. Neuroscience and Biobehavioral Reviews, 28, 317-32.

Corr, P. J. (2008). Reinforcement Sensitivity Theory (RST): Introduction. In P. J. Corr (Ed.), The Reinforcement Sensitivity Theory of Personality. New York, NY: Cambridge University Press.

Costa, P. T., \& McCrae, R. R. (1992). Neo Personality Inventory Revised (NEO PI-R). Psychological Assessment Resources.

DeYoung, C. G., Hirsh, J. B., Shane, M. S., Papademetris, X., Rajeevan, N., \& Gray, J. R. (2010). Testing predictions from personality neuroscience: Brain structure and the big five. Mind, 21, 820-828. doi:10.1177/0956797610370159 Testing.

Frings, L., Schulze-Bonhage, A., Spreer, J., \& Wagner, K. (2009). Remote effects of hippocampal damage on default network connectivity in the human brain. Journal of Neurology, 256, 2021-9. doi:10.1007/s00415-009-5233-0

Gardini, S., Cloninger, C., Robert, \& Venneri, A. (2009). Individual differences in personality traits reflect structural variance in specific brain regions. Brain Research Bulletin, 79, 265-70. doi:10.1016/j.brainresbull.2009.03.005

Good, C. D., Johnsrude, I. S., Ashburner, J., Henson, R. N., Friston, K. J., \& Frackowiak, R. S. (2001). A voxel-based morphometric study of ageing in 465 normal adult human brains. NeuroImage, 14, 21-36. doi:10.1006/nimg.2001.0786

Gray, J. (1977). Drug effects on fear and frustration: possible limbic site of action of minor tranquilizers. In L. Iversen, S. Iversen, \& S. Snyder (Eds.), Handbook of Psychopharmacology (Drugs,
Neurotransmitters and Behaviour, Vol. 8, pp. 433-529). New York, NY: Plenum Press.

Gray, J. (1982). The neuropsychology of anxiety: An enquiry into the functions of the septo-hippocampal system. Oxford, England: Oxford University Press.

Gray, J., \& McNaughton, N. (2000). The neuropsychology of anxiety: An enquiry into the functions of the septo-hippocampal system. Oxford, England: Oxford University Press.

Gur, R. C., Gunning-Dixon, F., Bilker, W. B., \& Gur, R. E. (2002). Sex differences in temporo-limbic and frontal brain volumes of healthy adults. Cerebral Cortex, 12, 998-1003.

Haas, B. W., Omura, K., Constable, R. T., \& Canli, T. (2007). Emotional conflict and neuroticism: Personality-dependent activation in the amygdala and subgenual anterior cingulate. Behavioral Neuroscience, 121, 249-56. doi:10.1037/0735-7044.121.2.249

Hahn, A., Stein, P., Windischberger, C., Weissenbacher, A., Spindelegger, C., Moser, E., Kasper, S., et al. (2011). Reduced resting-state functional connectivity between amygdala and orbitofrontal cortex in social anxiety disorder. NeuroImage, 56, 881-9. doi:10.1016/ j.neuroimage.2011.02.064

Hayano, F., Nakamura, M., Asami, T., Uehara, K., Yoshida, T., Roppongi, T., Otsuka, T., et al. (2009). Smaller amygdala is associated with anxiety in patients with panic disorder. Psychiatry and Clinical Neurosciences, 63(3), 266-76. doi:10.1111/j.1440-1819.2009.01960.x

Holland, P. C., \& Gallagher, M. (2004). Amygdala-frontal interactions and reward expectancy. Current Opinion in Neurobiology, 14, $148-55$.

Hu, X., Erb, M., Ackerman, H., Martin, J. A., Grodd, W., \& Reiterer, S. M. (2010). Voxel-based morphometry studies of personality: Issue of statistical model specification - effect of nuisance covariates. NeuroImage, 54, 1994-2005. doi:10.1016/ j.neuroimage.2010.10.024

Hundt, N., Nelson-Gray, R., Kimbrel, N., Mitchell, J., \& Kwapil, T. (2007). The interaction of reinforcement sensitivity and life events in the prediction of anhedonic depression and mixed anxietydepression symptoms. Personality and Individual Differences, 43, 1001-1012. doi:10.1016/j.paid.2007.02.021

Iidaka, T., Matsumoto, A., Ozaki, N., Suzuki, T., Iwata, N., Yamamoto, Y., Okada, T., et al. (2006). Volume of left amygdala subregion predicted temperamental trait of harm avoidance in female young subjects. A voxel-based morphometry study. Brain Research, 1125(1), 85-93. doi:10.1016/j.brainres.2006.09.015

Jackson, C. J. (2009). Jackson-5 scales of revised Reinforcement Sensitivity Theory (r-RST) and their application to dysfunctional real world outcomes. Journal of Research in Personality, 43, 556-569. doi:10.1016/j.jrp. 2009.02.007

Kasch, K. L., Rottenberg, J., Arnow, B. A., \& Gotlib, I. H. (2002). Behavioral activation and inhibition systems and the severity and course of depression. Journal of Abnormal Psychology, 111, 589597. doi:10.1037//0021-843X.111.4.589

Kim, M. J., \& Whalen, P. J. (2009). The structural integrity of an amygdala-prefrontal pathway predicts trait anxiety. Journal of Neuroscience, 29, 11614-8. doi:10.1523/JNEUROSCI.233509.2009

Kimbrel, N. A. (2008). A model of the development and maintenance of generalized social phobia. Clinical Psychology Review, 28, 592-612. doi:10.1016/j.cpr.2007.08.003

Kumari, V., Ffytche, D. H., Das, M., Wilson, G. D., Goswami, S., \& Sharma, T. (2007). Neuroticism and brain responses to anticipatory fear. Behavioral Neuroscience, 121, 643-52. doi:10.1037/ 0735-7044.121.4.643

Lacerda, A. L. T., Keshavan, M. S., Hardan, A. Y., Yorbik, O., Brambilla, P., Sassi, R. B., Nicoletti, M., et al. (2004). Anatomic evaluation of the orbitofrontal cortex in major depressive disorder. Biological Psychiatry, 55, 353-8. doi:10.1016/ j.biopsych.2003.08.021 
Maldjian, J. A., Laurienti, P. J., Kraft, R. A., \& Burdette, J. H. (2003). An automate method for neuroanatomic and cytoarchitectonic atlas-based interrogation of fMRI data sets. NeuroImage, 19, $1233-1239$.

Mardaga, S., \& Hansenne, M. (2007). Relationships between Cloninger's biosocial model of personality and the behavioral inhibition/approach systems (BIS/BAS). Personality and Individual Differences, 42, 715-722. doi:10.1016/j.paid.2006.08.013

McNaughton, N., \& Corr, P. J. (2004). A two-dimensional neuropsychology of defense: Fear/anxiety and defensive distance. Neuroscience and biobehavioral reviews, 28, 285-305. doi:10.1016/ j.neubiorev.2004.03.005

Montag, C., Buckholtz, J. W., Hartmann, P., Merz, M., Burk, C., Hennig, J., \& Reuter, M. (2008). COMT genetic variation affects fear processing: Psychophysiological evidence. Behavioral Neuroscience, 122, 901-9.

Oldfield, R. C. (1971). The assessment and analysis of handedness: The Edinburgh inventory. Neuropsychologia, 9, 97-113.

Phan, K. L., Fitzgerald, D. A., Nathan, P. J., \& Tancer, M. E. (2006). Association between amygdala hyperactivity to harsh faces and severity of social anxiety in generalized social phobia. Biological Psychiatry, 59, 424-9. doi:10.1016/j.biopsych.2005.08.012

Pinto-Meza, A., Caseras, X., Soler, J., Puigdemont, D., Perez, V., \& Torrubia, R. (2006). Behavioural Inhibition and Behavioural Activation Systems in current and recovered major depression participants. Personality and Individual Differences, 40, 215-226. doi:10.1016/j.paid.2005.06.021

Rauch, S. L., Shin, L. M., \& Wright, C. I. (2003). Neuroimaging studies of amygdala function in anxiety disorders. Annals of the New York Academy of Sciences, 985, 389-410.

Roppongi, T., Nakamura, M., Asami, T., Hayano, F., Otsuka, T., Uehara, K., Fujiwara, A., et al. (2010). Posterior orbitofrontal sulcogyral pattern associated with orbitofrontal cortex volume reduction and anxiety trait in panic disorder. Psychiatry and Clinical Neurosciences, 64, 318-26.

Scorzin, J. E., Kaaden, S., Quesada, C. M., Müller, C.-A., Fimmers, R., Urbach, H., \& Schramm, J. (2008). Volume determination of amygdala and hippocampus at 1.5 and 3.0T MRI in temporal lobe epilepsy. Epilepsy Research, 82, 29-37. doi:10.1016/ j.eplepsyres.2008.06.012

Smits, D. J. M., \& Boeck, P. D. (2006). From BIS/BAS to the big five. European Journal of Personality, 20, 255-270.

Sobanski, T., Wagner, G., Peikert, G., Gruhn, U., Schluttig, K., Sauer, H., \& Schlösser, R. (2010). Temporal and right frontal lobe alterations in panic disorder: A quantitative volumetric and voxel-based morphometric MRI study. Psychological Medicine, 40, 1879-1886.
Spampinato, M. V., Wood, J. N., De Simone, V., \& Grafman, J. (2009). Neural correlates of anxiety in healthy volunteers: A voxel-based morphometry study. Journal of Neuropsychiatry and Clinical Neurosciences, 21, 199-205. doi:10.1176/appi.neuropsych.21.2.199

Sportel, B. E., Nauta, M. H., de Hullu, E., de Jong, P. J., \& Hartman, C. A. (2011). Behavioral inhibition and attentional control in adolescents: Robust relationships with anxiety and depression. Journal of Child and Family Studies, 20, 149-156. doi:10.1007/s10826010-9435-y

Torrubia, R., Ávila, C., \& Caseras, X. (2008). Reinforcement Sensitivity Scales. In P. J. Corr (Ed.), The Reinforcement Sensitivity Theory of Personality. Cambridge, England: Cambridge University Press.

Torrubia, R., Ávila, C., Molto, J., \& Caseras, X. (2001). The Sensitivity to Punishment and Sensitivity to Reward Questionnaire (SPSRQ) as a measure of Gray's anxiety and impulsivity dimensions. Personality and Individual Differences, 31, 837-862.

Tzourio-Mazoyer, N., Landeau, B., Papathanassiou, D., Crivello, F., Etard, O., Delcroix, N., \& Joliot, M. (2002). Automated anatomical labeling of activations in SPM using a macroscopic anatomical parcellation of the MNI MRI single-subject brain. Neuroimage, 15, 273-89.

Van Schuerbeek, P., Baeken, C., De Raedt, R., De Mey, J., \& Luypaert, R. (2011). Individual differences in local gray and white matter volumes reflect differences in temperament and character: A voxel-based morphometry study in healthy young females. Brain Research, 1371, 32-42. doi:10.1016/j.brainres.2010.11.073

Vervoort, L., Wolters, L. H., Hogendoorn, S. M., de Haan, E., Boer, F., \& Prins, P. J. M. (2010). Sensitivity of Gray's Behavioral Inhibition System in clinically anxious and non-anxious children and adolescents. Personality and Individual Differences, 48, 629-633. doi:10.1016/j.paid.2009.12.021

Welborn, B. L., Papademetris, X., Reis, D. L., Rajeevan, N., Bloise, S. M., \& Gray, J. R. (2009). Variation in orbitofrontal cortex volume: Relation to sex, emotion regulation and affect. Social Cognitive and Affective Neuroscience, 4, 328-39. doi:10.1093/scan/nsp028

Wright, C. I., Williams, D., Feczko, E., Barrett, L. F., Dickerson, B. C., Schwartz, C. E., \& Wedig, M. M. (2006). Neuroanatomical correlates of extraversion and neuroticism. Cerebral Cortex, 16, 1809-19. doi:10.1093/cercor/bhj118

Yamasue, H., Abe, O., Suga, M., Yamada, H., Inoue, H., Tochigi, M., Rogers, M., et al. (2008). Gender-common and -specific neuroanatomical basis of human anxiety-related personality traits. $\mathrm{Ce}$ rebral Cortex, 18, 46-52. doi:10.1093/cercor/bhm030

Zald, D. H., \& Kim, S. W. (1996). Anatomy and function of the orbital frontal cortex, I: Anatomy, neurocircuitry, and obsessive-compulsive disorder. Journal of Neuropsychiatry and Clinical Neurosciences, 8 , 125-138. 